\title{
Drainage basins serve as multiple glacial refugia for alpine habitats in the Sierra Nevada Mountains, California
}

\author{
Yi-Ming Weng ${ }^{1}$, David Kavanaugh ${ }^{2}$, and Sean Schoville ${ }^{3}$ \\ ${ }^{1}$ University of Wisconsin Madison Graduate School \\ ${ }^{2}$ California Academy of Sciences \\ ${ }^{3}$ University of Wisconsin Madison
}

September 10, 2020

\begin{abstract}
The evolutionary histories of alpine species are often directly associated with responses to glaciation. Deep divergence among populations and complex patterns of genetic variation have been inferred as consequences of persistence within glacier boundaries (i.e. on nunataks), while shallow divergence and limited genetic variation is assumed to result from expansion from large refugia at the edge of ice shields (i.e. massifs de refuge). However, for some species, dependence on specific microhabitats could profoundly influence their spatial and demographic response to glaciation, and such a simple dichotomy may obscure the localization of actual refugia. In this study, we use the Nebria ingens complex (Coleoptera: Carabidae), a water-affiliated ground beetle lineage, to test how drainage basins are linked to their observed population structure. By analyzing mitochondrial COI gene sequences and genome-wide single nucleotide polymorphisms, we find that the major drainage systems of the Sierra Nevada Mountains in California best explain the population structure of the N. ingens complex. In addition, we find that an intermediate morphotype within the $\mathrm{N}$. ingens complex is the product of historical hybridization of N. riversi and N. ingens in the San Joaquin basin during glaciation. This study highlights the importance of considering ecological preferences in how species respond to climate fluctuations and provides an explanation for discordances that are often observed in comparative phylogeographic studies.
\end{abstract}

\section{Introduction}

The geographical distribution and diversification patterns of alpine species are commonly associated with historical glaciation cycles (Wallis, Waters, Upton, \& Craw, 2016). During periodic glacial climate fluctuations in the Pliocene and Pleistocene epochs, alpine species diversified as a consequence of population isolation, punctuated demographic change, and distributional range shifts (Knowles, 2001; Jansson \& Dynesis, 2002; Schoville and Roderick, 2009). Lineage-specific patterns of genetic structure and genetic diversity are often linked to the location of glacial refugia and subsequent processes of recolonization after the retreat of glaciers (Schönswetter, Stehlik, Holderegger, \& Tribsch, 2005). For example, European alpine species that retain substantial genetic diversity in a heterogeneous pattern across the alpine zone have been associated with persistence in high-elevation ice-free refugia (i.e. nunataks) during glaciation (Stehlik, Blattner, Holderegger, \& Bachmann, 2002; Schneeweiss, \& Schoenswetter, 2011). More generally, species that possess deep and extensive lineage diversity are often inferred to have persisted in multiple refugia, where isolated populations undergo strong genetic drift due to the smaller population size (Schoville \& Roderick 2010; Homburg et al., 2013; Rovito \& Schoville 2017). In contrast, populations that show shallower genetic structure across alpine distributions are inferred to have persisted in large, interconnected populations outside of the ice shields during glacial periods (i.e. massifs de refuge, or periglacial refugia). These two scenarios, at their extremes referred to as 'nunatak survival' and 'massifs de refuge', have been tested as competing hypotheses in many different alpine species (Wachter et al., 2016; Rovito \& Schoville 2017; Kosiński, Sękiewicz, Walas, 
Boratyński, \& Dering, 2019; Pan, Hülber, Willner, \& Schneeweiss, 2020). Both hypotheses are supported in different study systems, with emerging genomic datasets often favoring more complex population histories (Lohse, Nicholls, \& Stone, 2011).

Although this dichotomy of glacial survival considers the refugia being either outside or within the boundary of glaciation, which seems plausible for many alpine species, it might be insufficient for reconstructing the location of glacial refugia for species that live in specific habitats (Theissinger et al., 2013). Many alpine species require specific habitat requirements that are unlikely to be commonly found within or at the edge of large ice sheets. For example, the discordance in population structure of two co-distributed sedge species from the Rocky Mountains has been associated with their different microhabitat preferences (Massatti \& Knowles 2014). Interestingly, microhabitat partitioning is not rare for alpine species due to strong environmental gradients and interspecific interactions (Gereben, 1995; Kleckova, Konvicka, \& Klecka, 2014; Kikvidze et al., 2015). Subtle differences in microhabitat preference could be responsible for the discordant phylogeographic histories (Alvarez et al., 2009), even in co-distributed, interacting species (DeChaine \& Martin, 2006). In addition, as specific microhabitat requirements further constrain the spatial extent of suitable habitat in glacial refugia, the population structure of microhabitat specialists tends to show deeper diversification (Schoville \& Roderick, 2010 ; Rovito \& Schoville, 2017). Therefore, species' microhabitat could be a principal driver of population structure and evolutionary history, and in this study, we test whether microhabitat usage of a cold-specialized alpine species shapes lineage diversity patterns.

To test this hypothesis, we examine the population history of alpine ground beetles in the Nebria ingens species complex (Coleoptera: Carabidae), because they possess the characteristics of microhabitat specialization, host independence, limited dispersal ability, and strong genetic differentiation (Schoville, Roderick, \& Kavanaugh, 2012). Members of the $N$. ingens complex are restricted to high-elevation riparian, waterfall and 'seep' habitat (formed by melting snow and glaciers), where they forage as generalist predators and scavengers (of mostly invertebrates). While their habitat utilization and distribution is not host dependent, they are patchily distributed due to their narrow habitat preferences and limited dispersal ability. Like other alpineNebria, members of the $N$. ingens complex has atrophied hind wings that impede flight, restricting gene flow and distributional range size (Kavanaugh 1985). In fact, the $N$. ingens complex is limited to the Sierra Nevada Mountains of California and provides an interesting case study of recent alpine speciation (Schoville et al., 2012). The deep divergence between $N$. ingens and N. riversi, estimated roughly around a million years ago, suggests divergence in allopatry, yet several populations with intermediate phenotypes, and discordant mitochondrial and nuclear data, imply a more complex evolutionary history within this species complex.

Here, we reexamine the evolutionary history of the Nebria ingenscomplex using more extensive population sampling and genome-wide genetic markers, to identify how glacial refugia and post-glacial recolonization affect contemporary genetic structure. As population structure is the product of both contemporary gene flow and historical divergence (Paun, Schönswetter, Winkler, Consortium, \& Tribsch, 2008), evolutionary history can be complicated to interpret. Conventionally, we can apply coalescent-based approaches on multilocus data to simultaneously estimate the demographic parameters such as effective population size, divergence time, and migration rate (Carling, Lovette, \& Brumfield, 2010; Qu et al., 2012; Rougemont \& Bernatchez, 2018). However, alpine species typically possess complex population structure that is difficult to model (due to the large number of free parameters) without strong simplifying assumptions (e.g. Rovito and Schoville, 2018). Here, we employ a hypothesis testing framework based on population structure models (Table 1 ) to test competing scenarios of 1) a single or a few core glacial refugia, 2) drainage-associated refugia, or 3) survival within the extent of the glacial ice sheets ('nunatak survival'). For example, one or a few ancestral populations are expected if the entire species complex shifted into periglacial refugia and remained interconnected during glaciation. The glacial survival hypothesis (nunatak) would be supported if ancestral populations and observed genetic diversity patterns are directly associated with known ice free areas within the glacier boundary (Rood, Burbank, \& Finkel, 2011e; Figure 1 ). Finally, persistence in drainage basins is proposed, as we expect ancestral populations of the $N$. ingenscomplex were dependent on these geographical features to obtain suitable microhabitats. 


\section{Materials and Methods}

\section{Study System and testing for premating barriers to gene flow}

In previous genetic work, Schoville et al. (2012) revealed that there are two major genetic lineages within the $N$. ingens complex associated with the allopatric species $N$. ingens and $N$. riversi, but morphologically and genetically intermediate populations suggested some gene flow. The morphologically intermediate populations were readily distinguished by color pattern, as they were dull green, whereas $N$. riversi was bright metallic green and $N$. ingenswas matte black. Here we use the same convention to group individuals into three morphotypes. In order to understand whether these morphotypes are capable of genetic exchange, we used a simple mating experiment to assess premating isolation among the three morphotypes $(N$. riversi , intermediate, and $N$. ingens ). Reduced copulation across the three morphotypes would indicate limited interspecific gene flow. In this experiment, samples from three populations (sites 1, 8, and 27;Figure $\mathbf{1}$, Table 2 ) were chosen to represent $N$. riversi, the intermediate morphotype, and $N$. ingens, respectively. However, due to conservation concerns regarding the small local population sizes of beetles in the field, our experiment was constrained by sample size to 10-20 beetles per population. All beetles were collected in July 2018, maintained at $\sim 5^{\circ} \mathrm{C}$ in a refrigerator with a mealworm diet prior to experiments, and all the trials were conducted within a week following field collection. For each trial, one male and one female were placed within a large yogurt cup $(10 \mathrm{~cm}$ in diameter and $4 \mathrm{~cm}$ in depth) for 30 minutes. All experimental combinations of morphotypes were conducted (for both males and females), including experiments within each morphotype as controls, to determine the rate of successful copulation. A successful copulation was defined as the male mounting the female, with direct observation of his genitalia penetrating the female. Unfortunately, subsequent attempts to rear the progeny from the crosses failed in the lab.

\section{Genetic Sampling, DNA extraction and Sequencing}

In this study, we included 566 beetles from 27 populations of the $N$. ingens complex in the Sierra Nevada, California (Figure 1 ). The sample beetles were collected with permits granted by Sequoia and Kings National Parks (Study\# SEKI-0091), Yosemite National Park (Study\# YOSE-00093), and the California Fish and Game Department (\#SC-006997). Genomic DNA from these samples was extracted using the QIAamp 96 DNA Blood Kit (QIAGEN) or DNeasy Blood \& Tissue Kits (QIAGEN) for both genotyping by sequencing (GBS) and mitochondrial COI gene sequencing. In addition to 219 samples from the study of Schoville et al. (2012), 104 beetles were selected to sequence an approximately 645 base pair region (after trimming the primer sequences) of the mitochondrial COI gene using the universal arthropod primers Jerry (5'-CAACATTTATTTTGATTTTTTGG-3') and Pat (5'-TCCAATGCA CTAATCTGCCATATTA-3') (Simon et al. 1994).). The resulting dataset of 323 COI gene sequences were aligned for downstream analyses. For nuclear genomic analyses, 381 samples were selected for genotyping by sequencing (GBS) (Elshire et al, 2011), with 3 individuals replicated. The restriction enzyme ApeKI was used to reduce the genome to an appropriate size distribution and 150 base pair single-end Illumina sequencing libraries were prepared by the Biotechnology Center of University of Wisconsin-Madison. All genetic data are deposited at NCBI (see Data Accessibility statement).

\section{Processing of Genomic Data}

Genome-wide single nucleotide polymorphisms (SNPs) were called from the GBS data using Stacks v1.48 (Catchen, Hohenlohe, Bassham, Amores, \& Cresko, 2013). To generate a large set of reference loci (ustacks), a minimum depth of three reads was used to construct stacks of loci and two mismatches were allowed between two stacks (alleles). To build the catalog of loci among individuals (cstacks), two mismatches were allowed between loci. The loci were then filtered using the following criteria: loci were removed if (1) present in fewer than five populations, or (2) present in fewer than $30 \%$ of the individuals in a population. Before calling genotypes, further criteria were used to remove loci. First, the loci where more than $75 \%$ of individuals had a confounded match (multiple loci match to single catalog locus in an individual), or with likelihood less than -8.0, were removed. Second, SNPs with an error rate higher than 0.1 were dropped, with the error rate determined by the three replicated individuals. This resulted in a semi-filtered dataset comprised of 311 
individuals and 21,238 SNPs. In order to reduce any potential confounding effects from linkage disequilibrium or selection among SNPs, we further filtered this dataset such that only one SNP with the least missing data was retained for each GBS locus, and SNPs were removed that violated Hardy-Weinberg equilibrium at the population level based on a p-value of 0.001. This finalfully-filtered dataset comprised 311 individuals and $12,498 \mathrm{SNPs}$, and is used for downstream analyses that require independent loci.

\section{Phylogenetic tree reconstruction}

For the mitochondrial COI dataset, the alignment of 323 sequences was used to construct a Bayesian phylogenetic tree using the software Beast2 (Bouckaert et al, 2014), with out-group sequences of $N$. purpurata , N. meanyi , N. vandykei , N. praedicta, and N. metallica downloaded from GenBank (accession numbers: MN346054, KU641246, KU641248, JQ711382, KU875540). The model HKY+G was selected as the best substitution model based on model tests conducted with the software MEGA v7 (Kumar, Stecher, \& Tamura, 2016). A clock rate for the COI gene was set to 0.0113 per site per million years based on the best available COI mutation rate estimate for beetles, in this case related carabid beetles (Andújar, Serrano, \& Gómez-Zurita, 2012). Ten million steps of a Markov chain Monte Carlo (MCMC) were run to estimate model parameters and trees, with samples drawn every 1000 steps. Two separate runs were conducted. The posterior samples were assessed for convergence using TRACER v1.7 (Rambaut, Drummond, Xie, Baele, \& Suchard, 2018) and each parameter value was assessed to ensure a high effective sample size $(>200)$. Additionally, a statistical parsimony cladistic analysis, or TCS network, was reconstructed using Popart-1.7 (Clement, Snell, Walker, Posada, \& Crandall, 2002; Leigh \& Bryant, 2015). Based on the divisions in the TCS network, haplotypes were assigned to northern or southern haplogroups.

A population tree based on genome-wide SNP data (12,498 SNPs,fully-filtered dataset) was constructed using maximum likelihood in the program IQtree version 1.6.12 (Nguyen, Schmidt, Von Haeseler, \& Minh, 2015). The substitution model GTR +ASC was selected and the branch support was estimated based on 1,000 bootstrap replicates. The output tree was then visualized and colored using FigTree v1.4.1 (Rambaut, 2012). Additionally, we inferred the species tree using the program SNAPP (Bryant, Bouckaert, Felsenstein, Rosenberg, \& RoyChoudhury, 2012), implemented in BEAST2 version 2.6.2. Thefully-filtered dataset was used, but due to computational constraints, one random individual was selected from each of eight populations. Individuals were chosen from populations with relatively pure ancestry coefficients to avoid violating assumptions in the species tree approach, namely the effect of gene flow from other populations (see the result of sNMF analysis; Bryant et al., 2012, Stoltz et al., 2019). SNAPP was run for 30 million Markov chain Monte Carlo (MCMC) generations, with the custom program SNAPPER employed to run SNAPP at faster speed (Stoltz et al., 2019). Two independent runs were conducted and the output trees were visualized after 10\% burnin using DensiTree version 2.01 (Bouckaert 2010).

\section{Estimate of Ancestry Coefficients and Population Structure}

Sparse non-negative matrix factorization (sNMF) from the R package LEA v1.4.0 (Frichot, Mathieu, Trouillon, Bouchard, \& François, 2014) was used to reconstruct ancestry coefficients among sampled individuals in the fully-filtered dataset. To evaluate the most likely number of ancestral populations (K), we computed the cross-entropy criterion for $K=1$ to $K=20$ with ten replicates each. Since the lowest cross-entropy was not clear, we present multiple levels of $\mathrm{K}$ to interpret the ancestry coefficients (see results). The summed ancestry coefficients for each population were plotted in geographical pie charts to visualize geographical population structure (Figure S1 ).

\section{Testing spatial clustering by genetic cline}

To test whether the genetic clusters from SNMF are the consequence of natural genetic clusters or isolation by distance (IBD) with discontinuous sampling, we used the $\mathrm{R}$ package conStruct version 1.0.4 (Bradburd, Coop, \& Ralph, 2018). We compare the non-spatial (cluster) and spatial (IBD) models for $\mathrm{K}=1$ to $\mathrm{K}=10$ ancestral populations. The input allele frequencies of SNPs from fully-filtered dataset were calculated using VCFtools version 0.1.17 (Danecek et al., 2011), and the geographical distances among populations were calculated using R package geodist version 0.0.4 (Karney, 2013). In the conStruct package, we ran cross- 
validation analysis through $\mathrm{K}=3$ to $\mathrm{K}=10$ with ten replicates and 100,000 iterations of the MCMC chains for each K. The predictive accuracies were estimated with $90 \%$ training and $10 \%$ test data. Two-sample t-tests were used to test differences between spatial and non-spatial models for each K. Moreover, we also consider the preferred models by examining the layer contributions from $K=1$ to $K=10$ using the construct function, with two MCMC chains of 100,000 iterations (Bradburd, Coop, \& Ralph, 2018).

\section{Estimate of demographic history}

In order to better understand the demographic history of the $N$. ingens complex, we employed Stairway plot version 2 ( Liu and Fu 2015, Liu 2020) to reconstruct the historical demography of focal populations using the semi-filtered dataset. Similar to SNAPP, the eight populations with pure ancestry coefficients were used to minimize any bias of recent gene flow. The input folded site allele frequency spectrum was estimated for each population using the custom program easySFS (https://github.com/isaacovercast/easySFS). We used a point mutation rate of $2.8 \times 10^{-9}$ per site per generation, which was estimated based on Drosophila and is close to the nuclear mutation rate of other non-social insects (Keightley, Ness, Halligan, \& Haddrill, 2014, Keightley et al., 2015, Oppold and Pfenninger 2017). Other parameters were set to default.

\section{Testing refugia models with TMRCA of paired populations}

The inferred ancestral clusters from sNMF and conStruct were further tested as refugial populations by comparing the divergence times of paired populations. Under a drainage refugia hypothesis, we expect to find older divergence times between populations from different drainages systems than within drainage systems, as the two populations retreated to separate glacial refugia and were isolated for a longer period of time. For the preferred ancestral clustering model $(\mathrm{K}=6)$, we assigned populations to major drainage basins in the Sierra Nevada based on the locations of their collection sites (Table 2 ). If populations had nearly equal distance from two different drainages, they were assigned to both drainages. As several alternative ancestral clustering models $(\mathrm{K}=4$ and $\mathrm{K}=5)$ from $\mathrm{sNMF}$ were difficult to distinguish from $\mathrm{K}=6$, we examined the effect of this analysis under these alternative clustering models (Figure S1, Table $\mathbf{1}$ ). We grouped populations from Tuolumne and Merced drainages into the same refugium $(\mathrm{K}=5)$, and further grouped populations from the Kern and Kaweah drainages into single refugium $(K=4)$. Finally, we considered a three refugia model, where populations were grouped within the same morphotype.

Paired populations were chosen by grouping collecting sites with their nearest four to seven geographical neighbors, including sites from the same and/or different drainages or morphotypes. The time of most recent common ancestor (TMRCA) of the paired populations was estimated using the joint site frequency spectrum in סaoi version 2.0.3 (Gutenkunst, Hernandez, Williamson, \& Bustamante, 2009). Since there is a potential bias in using filtered genotype data (Warmuth and Ellegren 2019), we estimated the two-dimensional allele frequency spectrums (2d-sfs) directly from the GBS reads. The 2d-sfs estimation from GBS reads requires a reference genome, so we generated a de novo assembly of $N$. ingens from a single male individual using 130x coverage Illumina NovaSeq pair-end reads. We used SPAdes version 3.14.1 (Bankevich et al., 2012) to assemble the genome (more details regarding genome assembly are described in the Supplementary Methods). The GBS reads were mapped to the reference genome using STAR version 2.7.3a and used to calculate the optimized 2d-sfs using angsd version 0.931, after filtering out the low quality reads using default quality control settings (Dobin et al., 2013, Korneliussen, Albrechtsen, \& Nielsen, 2014). The TMRCA, as well as the migration rate and effective population size of each population, were approximated with the $2 \mathrm{~d}$-sfs using dadi_pipeline version 3.1.4 (Gutenkunst et al., 2009, Portik et al., 2017). In the dadi_pipeline program, we selected the divergence with continuous symmetric migration model and ran this 10 times in a 4-run (10, 20, 30, 40 replications) or 5-run (10,20,30, 40, 50 replications) optimization, with grid size starting from the number that was rounded up from the larger allele size to the nearest ten, until the likelihoods and optimized parameters reached a stable state. The parameters from the optimization with highest likelihood score were selected to represent the best optimization. The standard deviations of each set of parameters was estimated using 100 nonparametric bootstraps of the $2 \mathrm{~d}$-sfs generated with $\delta$ aoi $\mathrm{i}$, and then the Godambe Information Matrix (GIM) was used to analyze the uncertainty of the parameters based on the 100 bootstrap 2d-sfs (Coffman, Hsieh, Gravel, \& Gutenkunst, 2016). 
The TMRCA estimations of paired populations were used as dependent variable, with grouping status (from the same or different drainage, cluster, or morphotype) used as a categorical independent variable and geographical distance as a covariate in an analysis of covariance (ANCOVA). For each ANCOVA regression model, AIC and BIC were calculated, and Vuong's test was used to compare those models using the R package nonnest2 version 0.5-5 (Vuong, 1989; Merkle \& You, 2018).

\section{Testing effects of post glacial population structure}

Compared to the estimation of divergence time, the fixation index $F$ ST is more sensitive to gene flow among populations, where paired populations experiencing gene flow are expected to have lower $F$ st values (in a 0 to 1 scale). Therefore, if gene flow occurred across drainage basins after populations recolonized high elevation areas (i.e. across mountain ridges), we expect to see little to no discrepancy in comparisons of populations from the same or different watersheds. For the same set of population pairs of the best model in the TMRCA analysis, we calculated the means of Weir \& Cockerham's $F$ ST in VCFtools version 0.1.17 (Weir \& Cockerham 1984, Danecek et al., 2011) using thesemi-filtered dataset. Similar to the TMRCA, the ANCOVA was used to test whether population pairs from different drainages show higher $F$ sT, with geographic distance between paired populations as a covariate.

\section{Results}

\section{Mating trials}

Mating trials demonstrated that each morphotype within the $N$. ingens complex could successfully copulate with its own morphotype and with other morphotypes (Table $\mathbf{3}$ ). While we observed a reduction in successful mating in experiments involving $N$. ingens, this also occurred when $N$. ingens males were paired with $N$. ingens females, suggesting that it may have been linked to reproductive status (e.g . reproductive diapause). Successful copulation occurred across all combinations of morphotypes, although not always for both male and female directed crosses. We note, however, that sample sizes are small.

\section{Mitochondrial phylogeny}

The COI phylogenetic tree has two major lineages, a northern and southern clade with divergence time of 0.52 million years ago, and both clades contain individuals from the intermediate morphotype (Figure 2 ). There is clear geographical structure, with populations from the same drainage belonging to the same clades and even the same haplotype. For example, there is a distinct subclade in the northern clade that includes samples from the Tuolumne and Merced drainages, and excludes other haplotypes found in the northern clade. Similarly, several clades in the southern lineage consist of samples only from the Kern or Kern/Kaweah drainage. The TCS network shows that the two distinct haplogroups are separated by eight substitutions (Figure S2 ). Haplotypes assigned to the northern clade are widely distributed, although many are unique and occur locally. These haplotypes are found in the Tuolumne ( $N$. riversi ), San Joaquin (intermediate morphotype), Kings, and Kern ( N. ingens ) drainages, including 13 out of the 21 populations of intermediate and $N$. ingens sample sites, as far as the southernmost population (site27). On the other hand, the southern clade consists only of samples from San Joaquin (intermediate morphotype), Kings, Kern, and Kaweah ( $N$. ingens ), and no southern clade haplotypes are observed in northern populations of N. riversi . (Table $\mathbf{S} 1$ ).

\section{Nuclear Phylogeny}

The species tree inferred using SNAPP, based on individuals from eight sample sites, also shows two major clades. However, a northern clade includes only samples from the Tuolumne, Merced, and San Joaquin drainages, whereas a southern clade only includes samples from Kings, Kaweah, and Kern drainages (Figure 3 ). The species tree topology has strong posterior branch support for all nodes, and suggests that morphologically intermediate populations from the San Joaquin watershed are genetically closer to the northern $N$. riversiindividuals. In contrast, the concatenated Maximum likelihood analysis using IQtree does not show two major clades, but instead represents the evolutionary divergence as hierarchically nested branches of populations that reflect geographical proximity (Figure 4 ). Bootstrap support is high for populations, 
though weak for a few branches joining different populations. It is also worthwhile to note that the tree topology largely supports a clear geographical subdivision by drainage system (Figure 4 ).

Population Structure and Ancestry Coefficients

The clustering of individuals into ancestral populations was estimated with sNMF using cluster numbers from 1 to 20. The optimal number of clusters was unclear as the cross-entropy continuously decreases up to $\mathrm{K}=11$, although the relative change in cross-entropy values is smaller after $\mathrm{K}=6$ (Figure S3 ). In the $\mathrm{K}=3$ model, clusters are consistent with morphotypes ( $N$. riversi, intermediate, and $N$. ingens $)$, but with some populations showing some admixture among these major lineages. These admixed populations are located in a geographical transition zone where separate lineages could have experienced historical gene flow (Figure S1 ). In the $\mathrm{K}=4$ model, the southern cluster splits into two clusters, revealing a distinct ancestral subpopulation located in the Kings drainage. In the $\mathrm{K}=5$ model, the southernmost cluster further subdivides into west (Kaweah) and east (Kern) ancestral populations. In the $\mathrm{K}=6$ model, the northern sample sites (sites 3 and 4) split from other northern populations and thus subdivide $N$. riversi (sites 1 to 6) into two clusters. From $\mathrm{K}=7$ to $\mathrm{K}=11$, new clusters mostly emerge around the contact zones of major drainages (Figure S1 ).

\section{Testing spatial clustering by genetic cline}

Comparisons between spatial and non-spatial models using conStruct reveal that the spatial model is always favored (Figure 5), as the accuracies of the spatial model are significantly higher than that of non-spatial model in $K=1$ to $K=8$. In addition, the analysis of layer contributions for $K=1$ to $K=10$ shows that additional layers contribute very little at $\mathrm{K}=5$ and become negligible at $\mathrm{K}=7$ (FigureS4 ). Viewed in combination with the results from $\mathrm{sNMF}, \mathrm{K}=4$ to $\mathrm{K}=6$ are preferred models.

\section{Estimate of demographic history}

In the analyses of demographic change, the stairway plots of the eight populations show similar patterns, with population size declining dramatically from around 100 thousand to a few thousands individuals within the last 30 thousand years (Figure 6 ). According to the stairway plots, the sample sites 1 and 3 (N. riversi ) have similar patterns of population decline starting around 25 thousand years ago, but the ancestral population was larger in site 3, a site located in the contact zone of the Merced and San Joaquin drainage systems (Figure 6A \& B ). The sites 9, 10, and 12 represent intermediate morphotypes located within San Joaquin drainage, and they share similar patterns of decline starting around 10 thousand years ago (Figure 6C, D, \&E ). The three sites of $N$. ingens, from the Kings (site 17), Kaweah (site 24), and Kern (site 27), shows declines ranging from 20 thousand to two thousand years ago (Figure $6 \mathbf{F}, \mathbf{G}, \mathbf{\& H}$ ).

\section{Testing glacial refugia and post glacial recolonization}

The TMRCA estimation of 96 pairs of populations were used to conduct ANCOVA for models of $\mathrm{K}=3$ to $\mathrm{K}=6$. In all tested models, the interaction terms are not significant and thus the type II sum of squares was used in ANCOVA tests. The $\mathrm{K}=6$ drainage refugia model includes 30 pairs of populations from the same drainage system and 66 pairs of populations from different drainage systems. This model has the lowest AIC and BIC, and explains the data significantly better than either $\mathrm{K}=3$ or $\mathrm{K}=4$ population structure models based on Vuong's test (Table 1 ). However, we cannot distinguish the $\mathrm{K}=5$ and $\mathrm{K}=6$ population structure model, as they possess the same set of paired populations due to the lack of multiple population samples form the Merced drainage.

In the best fit $\mathrm{K}=6$ model, the result of ANCOVA shows that TMRCA estimates are significantly older (> 20,000 years ago) for pairs of populations from different drainages than from the same drainage $(\sim 10,000$ years ago; p-value $<0.001)$, regardless of whether geographical distance is included as a covariant or not (Figure 7A ). However, there are a few of population pairs from the same drainage that do have older TMRCA, notably sites in the Kings or Kern drainages. Similarly, there are a few outliers with recent TMRCA when pairs of different drainages are compared, including sites from the San Joaquin and Kings drainages. In contrast, there is no significant difference between the two types of population pairs when 
measuring Weir and Cockerham's $F$ st (p-value $>0.05)$. Geographical distance has a high $R^{2}$ value $(0.35$ and 0.55 ) when included as a covariate, implying genetic variation is consistent with isolation by distance (Figure 7 B).

\section{Discussion}

The main goal of this study was to test competing hypotheses for the location of glacial refugia in an alpine beetle species, including a hypothesis focusing on the role of drainage basins as refugia. By focusing on analyses of population structure and TMRCA estimates from paired populations of the cold-specialized, riparian beetles in the alpine $N$. ingenscomplex, we found strong evidence that population structure and patterns of lineage divergence are correlated with drainage basins in the Sierra Nevada Mountains, California. This supports the hypothesis that drainage basins, and by extension microhabitat preferences of a species, allow for persistence in multiple refugia across the mountain range. We also provide evidence that postglacial gene flow can readily obscure the signature of glacial refugia, if not carefully dissected, and that episodes of glaciation enhanced lineage diversity in this alpine species complex through both isolation and hybridization.

\section{Disentangling sources of population structure}

The population structure of a species reflects both historical divergence processes and contemporary gene flow among subpopulations, and it requires careful analysis to disentangle these evolutionary processes (Nielsen, \& Slatkin, 2007; Schönswetter \& Schneeweiss 2019). Summary statistics, such as Wright's $F$ ST, are useful for describing genetic drift and gene flow under equilibrium models, but provide little information about deeper evolutionary events that shape patterns of genetic variation. Clustering based algorithms, which infer individual ancestry components according to a model, are also challenging to interpret when population divergence is continuous in space (François \& Durand, 2010; but see Bradburd et al. 2018). Coalescent approaches leverage the distribution of gene genealogies to separately estimate the population parameters, including migration rate and divergence time, and can be used to distinguish models of migration from divergence (Nielsen \& Wakeley, 2001). However, coalescent approaches are often difficult to implement when evolutionary history is spatially complex, as the number of free parameters grows rapidly (Beerli, 2009).

In this study, we leveraged aspect of each approach to disentangle the evolutionary history of the $N$. ingens complex. First, we used the clustering algorithm sNMF to identify population structure, though we found that the log likelihood increased with an increasing number of clusters. A large shift in K occurred as different morphotypes were distinguished genetically $(\mathrm{K}=3)$, but models $\mathrm{K}=4$ to $\mathrm{K}=6$ had improved log likelihood scores and revealed further population subdivision reflecting major drainage systems in the Sierra Nevada. More subtle patterns of admixture among drainages continued to emerge as the number of clusters increases (Figure S3 ), but this appears to be the product of gene flow and uneven sampling (continuous population structure). Indeed, by explicitly comparing spatial and non-spatial cluster models using conStruct, we found that the spatial models are generally more accurate than the non-spatial models (Figure $\mathbf{5}$ ), indicating the presence of isolation by distance among sample sites. Analysis of pairwise $F$ sT values among populations also strongly supports gradual genetic divergence with geographical distances $\left(R^{2}=0.35\right.$; Figure 7$)$.

We further assessed patterns of population divergence using a model fitting framework based on coalescence theory (Rougemont \& Bernatchez, 2018). We compared ANCOVA regressions on the paired populations' TMRCA for different levels of population structure. For TMRCA, $\mathrm{K}=6$ and $\mathrm{K}=5$ show the best AIC, BIC, and results from the Vuong's test. Although $\mathrm{K}=6$ and $\mathrm{K}=5$ are indistinguishable owing to the lack of multiple population samples from the Merced drainage, both support the model of drainage associated glacial refugia (Table 1 ).

\section{Differentiating drainage based multiple refugia from nunatak refugia}

Although our results suggest multiple ancestral populations and high levels of genetic diversity in some high elevation populations, which could fit the predictions of glacial survival within the ice sheet (nunatak survival hypothesis), the pattern of several isolated glacial refugia of $N$. ingens complex is fundamentally different from 
nunataks. Conventionally, nunatak refugia refer to the small, ice-free geographical areas within the glacial ice sheets. In such habitats, populations would be restricted to small size and exposed to extremely challenging environmental conditions (cold and windy, and thus xeric), before expanding in postglacial periods (Dahl 1987; Holderegger \& Thiel-Egenter 2009). In the case of the $N$. ingens complex, high elevation sites with elevated genetic diversity reflect recent admixture among ancestral population clusters (conStruct results, seeTable 1 ). Ancestral populations are not associated with ice-free areas in the north and central part of the Sierra Nevada (Figure 1 ). Similar contact zones are found in a large set of alpine plants from the European Alps (Thiel-Egenter et al., 2011), and a recent synthesis of alpine studies suggests this might be a common phenomenon across long linear mountain ranges (Wallis et al., 2016). Many alpine or sub-alpine species that have patterns consistent with multiple glacial refugia are certainly not related to persistence in nunataks (Kubow, Robinson, Shama, \& Jokela, 2010; Homburg et al., 2013), suggesting that the deep divergence of population structure or multiple ancestral populations might not be sufficient to be directly linked to nunatak refugia.

The other common character of populations recolonized from the nunataks is the vestige of a bottleneck during glacial periods (Segarra-Moragues, Palop-Esteban, Gonzalez-Candelas, \& Catalan, 2007; Kosiński et al., 2019). Our data suggest the opposite has happened, as the stairway plots show drastic population decline initiating at the last glacial maximum and accelerating into the present (Figure 7 ). Similarly, estimates of ancestral population size (reference population, $\mathrm{N}_{\text {ref }}$ ) in $\delta$ aoi were several fold larger than the descendant populations (Table S2 ). Although other studies report glacial bottlenecks or postglacial population increases (Bidegaray-Batista et al., 2016; Sim, Hall, Jex, Hegel, \& Coltman, 2016; Starcová, Vohralík, Kryštufek, Bolfíková, \& Hulva, 2016; Weng, Yang, \& Yeh, 2016; Huang et al., 2017), it seems logical that alpine species would undergo founder events as they recolonize high elevation areas following glacier retreat (Schoville and Roderick, 2009).

We acknowledge that the continuous spatial structure of population could possibly lead to a false signature of recent population decline for stairway plot analysis and possibly other sfs-based analyses including oaoi (Battey, Ralph, \& Kern, 2020). However, there are several reasons we interpret the larger ancestral pop-

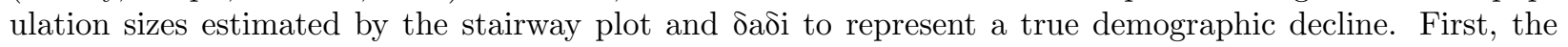
census population size of populations within the $N$. ingenscomplex is mostly very small and populations are isolated from each other by complex topographic terrain (e.g . suitable habitat occurs within the first few hundred meters from the headwaters of streams or small seep areas within glacier cirques). Thus, estimates of a contemporary effective population size numbering in the hundreds or several thousand is realistic in our opinion. Second, all eight sample sites show a consistent trend of population decline, even though they differ in their neighborhood size and geographical position in the geographical cline, which are major factors that bias the stairway plot estimates in continuously structured populations (Battey et al., 2020). Lastly, the cool and wet glacial period preceding the glacial maximum would likely be optimal for a cold-specialized, riparian beetle, and thus explains the larger population size at this time. A few other studies have shown population expansion during glaciation followed by postglacial population declines in alpine and subalpine species (Galbreath, Hafner, \& Zamudio, 2009; Huang et al., 2016; Zaman, Hubert, \& Schoville, 2019).

Divergence, admixture and speciation

The role of climate fluctuations in causing secondary contact of recently diverged species is well known (Hewitt 1999), with the outcomes varying from free admixture, to directional gene flow, to complete isolation or even hybrid speciation. Several cases demonstrating the range of these outcomes are known from alpine taxa within the Sierra Nevada Mountains (Gompert, Fordyce, Forister, Shapiro, \& Nice, 2006; Rovito 2010; Rubidge, Patton, \& Moritz, 2014). The degree and geographical scale of admixture likely varies across taxa due to a number of factors, including the dispersal ability of species, habitat suitability and connectivity, as well as the reproductive compatibility of lineages and fitness of hybrids (Barton \& Hewitt 1985; Dufresnes, Berroneau, Dubey, Litvinchuk, \& Perrin, 2020).

In this study, we found that an intermediate morphotype in the $N$. ingens complex (Schoville et al. 2012) extends to populations throughout the Central Sierra Nevada, encompassing the entire San Joaquin drainage 
and extending south to contact zones in the Kings River watershed (sites 14 and 15; Figure 1 ). The distribution of the morphological phenotype is different from the observed genetic patterns, where mitochondrial haplotype variation suggests introgression from $N$. riversi into nearly the entire southern range of $N$. ingens (Figure 2 \& S2 ), and genome-wide nuclear markers suggest that the intermediate morphotype is an independent lineage (site 9, 10 and 12; Figure 3 ). For example, in the sNMF analysis (from $\mathrm{K}=3$ to $\mathrm{K}=11$ ), most individuals representing the intermediate morphotype do not show extensive ancestry from either $N$. riversior $N$. ingens (Figure S1 ). Additionally, TMRCA estimates between populations of the intermediate morphotype and either species mostly predate the last glacial maximum. Despite this genetic divergence, we found no evidence of premating isolation among the three lineages (Table $\mathbf{3}$ ), although our sample sizes were low.

Based on these results, we infer that 1) an early glacial cycle caused deep divergence to form between northern and southern populations, which formed $N$. ingens and $N$. riversi . In a subsequent glacial cycle, 2) a contact zone formed near the San Joaquin drainage and led to the formation of the intermediate morphotype. This lineage diversity was maintained during the last glacial maximim and 3) allowed for the formation of more complex population structure as each major lineage recolonized high elevation habitat. It is clear that limited gene flow occurs in parts of the alpine range, leading to the formation of a genetic cline in genetic divergence measures such as $F$ sT. The general pattern we observe is consistent with models of diversification linked to the orbitally forced range dynamic (ORD) model (Dynesius \& Jansson, 2000), and the more recently coined 'mix-isolation-mix' model proposed by He et al. (2019).

\section{Implications for alpine species conservation}

Our finding of a drastic population decline since the last glaciation retreat, coupled with field observations of extremely limited habitat and small population sizes, suggests that this species complex may be vulnerable to localized extinction. For many Nebria species in North America, lower elevational distributions have shifted upward in the past 40 years (Kavanaugh 2015). If population range shifts are associated with warmer temperatures in the Holocene, it is very likely that populations will continue to shrink with ongoing global warming. This could be exacerbated by the loss of Sierra Nevada glaciers and a reduction in winter snowpack (Basagic \& Fountain, 2011; Belmecheri, Babst, Wahl, Stahle, \& Trouet, 2016). Although N. ingens complex is not an economically significant animal, it is difficult to predict the consequence of a general predator disappearing from a comparatively depauperate alpine ecosystem.

\section{Acknowledgements}

We are grateful to the National Parks of the United States including Yosemite, Sequoia, and King Canyon National Parks for providing permission to conduct research in the parks. Thanks also to Ministry of Education Republic of China (Taiwan) for offering a scholarship to YMW. This research is also supported by the Valentine Eastern Sierra Reserve (UC Santa Barbara Natural Reserve System) through VESR Graduate Student Funding to YMW, and a UW Research Competition Award to SDS. We thank Benton Veire and Matthew Medeiros for helping us to conduct the field work, and Ph.D. committees of YMW for providing suggestions to this study. The authors have no conflicts of interests to declare.

\section{References}

Alvarez, N., Thiel-Egenter, C., Tribsch, A., Holderegger, R., Manel, S., Schönswetter, P., Taberlet, P., Brodbeck, S., Gaudeul, M., Gielly, L., Küpfer, P., Mansion, G., Negrini, R., Paun, O., Pellecchia, M., Rioux, D., Schüpfer, F., Van Loo, M., Winkler, M., Gugerli, F., \& IntraBioDiv Consortium (2009). History or ecology? Substrate type as a major driver of spatial genetic structure in Alpine plants. Ecology Letters , 12(7), 632- 640 .

Andújar, C., Serrano, J. \& Gómez-Zurita, J. (2012). Winding up the molecular clock in the genus Carabus (Coleoptera: Carabidae): assessment of methodological decisions on rate and node age estimation. $B M C$ Evolutionary Biology , 12, 40.

Bankevich, A., S. Nurk, Antipov, D., Gurevich, A. A., Dvorkin, M., Kulikov, A. S., Lesin, V. M., Nikolenko, 
S. I., Pham, S., Prjibelski, A. D., Pyshkin, A. V., Sirotkin, A. V., Vyahhi, N., Tesler, G., Alekseyev, M. A., \& Pevzner, P. A. (2012). SPAdes: a new genome assembly algorithm and its applications to single-cell sequencing. Journal of Computational Biology , 19 , 455- 477.

Barton, N. H., \& Hewitt, G. M. (1985). Analysis of hybrid zones.Annual Review of Ecology and Systematics , $16,113-148$.

Basagic, H. J., \& Fountain, A. G. (2011). Quantifying 20th century glacier change in the Sierra Nevada, California. Arctic, Antarctic, and Alpine Research , 43(3) , 317- 330.

Battey, C. J., Ralph, P. L., \& Kern, A. D. (2020). Space is the place: effects of continuous spatial structure on analysis of population genetic data. Genetics , 215, 193- 214.

Beerli, P. (2015). How to use MIGRATE or why are Markov chain Monte Carlo programs difficult to use. Population Genetics for Animal Conservation, 17, 42- 79.

Belmecheri, S., Babst, F., Wahl, E. R., Stahle, D. W., \& Trouet, V. (2016). Multi-century evaluation of Sierra Nevada snowpack. Nature Climate Change, 6(1) , 2- 3.

Bidegaray-Batista, L., Sánchez-Gracia, A., Santulli, G., Maiorano, L., Guisan, A., Vogler, A. P., \& Arnedo, M. A. (2016). Imprints of multiple glacial refugia in the Pyrenees revealed by phylogeography and palaeodistribution modelling of an endemic spider. Molecular Ecology , 25, 2046- 2064.

Bohonak, A. J. (1999). Dispersal, gene flow, and population structure. The Quarterly Review of Biology , 74 , 21- 45 .

Bouckaert, R. R. (2010). DensiTree: making sense of sets of phylogenetic trees. Bioinformatics , 26 , $1372-$ 1373.

Bouckaert, R., Heled, J., Kühnert, D., Vaughan, T., Wu, C-H., Xie, D., Suchard, MA., Rambaut, A., \& Drummond, A. J. (2014). BEAST 2: A Software Platform for Bayesian Evolutionary Analysis. PLoS Computational Biology , 10(4) , e1003537. doi:10.1371/journal.pcbi.1003537

Bradburd, G., Coop, G., \& Ralph, P. (2018). Inferring continuous and discrete population genetic structure across space. Genetics, $210,33-52$.

Bryant, D., Bouckaert, R., Felsenstein, J., Rosenberg, N. A., \& RoyChoudhury, A. (2012). Inferring species trees directly from biallelic genetic markers: bypassing gene trees in a full coalescent analysis.Molecular Biology and Evolution, 29 , 1917- 1932.

Carling, M. D., Lovette, I. J., \& Brumfield, R. T. (2010). Historical divergence and gene flow: coalescent analyses of mitochondrial, autosomal and sex-linked loci in Passerina buntings .Evolution, 64: 1762- 1772.

Catchen, J., Hohenlohe, P. A., Bassham, S., Amores, A., \& Cresko, W. A. (2013). Stacks: an analysis tool set for population genomics. Molecular Ecology , 22, 3124- 3140.

Clement, M., Snell, Q., Walker, P., Posada, D., \& Crandall, K. (2002). TCS: estimating gene genealogies. Brigham Young University, Provo, UT.

Coffman, A. J., Hsieh, P. H., Gravel, S., \& Gutenkunst, R. N. (2016). Computationally Efficient Composite Likelihood Statistics for Demographic Inference. Molecular Biology and Evolution ,33(2) , 591- 593. https://doi.org/10.1093/molbev/msv255

Dahl, E. (1987). The nunatak theory reconsidered. Ecological Bulletins , 77-94.

Danecek, P., Auton, A., Abecasis, G., Albers, C. A., Banks, E., DePristo, M. A., Handsaker, R. E., Lunter, G., Marth, G. T., Sherry, S. T., McVean, G., Durbin, R., \& 1000 Genomes Project Analysis Group. (2011). The variant call format and VCFtools. Bioinformatics ,27, 2156- 2158. 
DeChaine, E. G., \& Martin, A. P. (2006). Using coalescent simulations to test the impact of Quaternary climate cycles on divergence in an alpine plant-insect association. Evolution , 60(5) , 1004- 1013.

Dobin, A., Davis, C. A., Schlesinger, F., Drenkow, J., Zaleski, C., Jha, S., Batut, P., Chaisson, M., \& Gingeras, T. R. (2013). STAR: ultrafast universal RNA-seq aligner. Bioinformatics , 29 , 15- 21.

Dufresnes, C., Berroneau, M., Dubey, S., Litvinchuk, S. N., \& Perrin, N. (2020). The effect of phylogeographic history on species boundaries: a comparative framework in Hyla tree frogs. Scientific Reports , 10 , $1-12$.

Dynesius, M., \& Jansson, R. (2000). Evolutionary consequences of changes in species' geographical distributions driven by Milankovitch climate oscillations. Proceedings of the National Academy of Sciences, 97(16) , 9115- 9120.

Elshire, R.J., Glaubitz, J. C., Sun, Q., Poland, J. A., Kawamoto, K., Buckler, E. S., \& Mitchell, S. E. (2011). A robust, simple genotyping-by-sequencing (GBS) approach for high diversity species.PLoS ONE , 6, e19379.

Francois, O. \& Durand, E. (2010). Spatially explicit Bayesian clustering models in population genetics. Molecular Ecology Resources , 10(5) , 773- 784

Frichot, E., Mathieu, F., Trouillon, T., Bouchard, G., \& Francois, O. (2014). Fast and efficient estimation of individual ancestry coefficients. Genetics , 196, 973-983.

Galbreath, K. E., Hafner, D. J., \& Zamudio, K. R. (2009). When cold is better: climate-driven elevation shifts yield complex patterns of diversification and demography in an alpine specialist (American pika,Ochotona princeps ). Evolution, 63 , 2848- 2863.

Gereben, B. A. (1995). Co-occurrence and microhabitat distribution of six Nebria species (Coleoptera: Carabidae) in an alpine glacier retreat zone in the Alps, Austria. Arctic and Alpine Research ,27(4), 371379.

Gompert, Z., Fordyce, J. A., Forister, M. L., Shapiro, A. M., \& Nice, C. C. (2006). Homoploid hybrid speciation in an extreme habitat.Science, 314(5807), 1923- 1925.

Gutenkunst, R. N., Hernandez, R. D., Williamson, S. H., \& Bustamante, C. D. (2009). Inferring the joint demographic history of multiple populations from multidimensional SNP frequency data. PLoS Genetics , 5, e1000695.

He, Z., Li, X., Yang, M., Wang, X., Zhong, C., Duke, N. C., Wu, C. I., \& Shi, S. (2019). Speciation with gene flow via cycles of isolation and migration: insights from multiple mangrove taxa. National Science Review , 6(2), 275- 288.

Hewitt, G. M. (1999). Post-glacial re-colonization of European biota.Biological Journal of the Linnean Society, 68, 87- 112 .

Holderegger, R., \& Thiel-Egenter, C. (2009). A discussion of different types of glacial refugia used in mountain biogeography and phylogeography. Journal of Biogeography, 36 , 476- 480.

Homburg, K., Drees, C., Gossner, M. M., Rakosy, L., Vrezec, A., and Assmann, T. (2013). Multiple glacial refugia of the low-dispersal ground beetle Carabus irregularis : molecular data support predictions of species distribution models. PLoS One, 8, e61185.

Huang, C. C., Hsu, T. W., Wang, H. V., Liu, Z. H., Chen, Y. Y., Chiu, C. T., Huang, C. L., Hung, K. H., \& Chiang, T. Y.. (2016). Multilocus analyses reveal postglacial demographic shrinkage of Juniperus morrisonicola (Cupressaceae), a dominant alpine species in Taiwan.PLoS One, 11, e0161713.

Huang, Z. S.,. Yu, F. L, Gong, H. S., Song, Y. L., Zeng, Z. G., \& Zhang, Q. (2017). Phylogeographical structure and demographic expansion in the endemic alpine stream salamander (Hynobiidae:Batrachuperus 
) of the Qinling Mountains. Scientific Reports, 7, 1871.

Jansson, R., \& Dynesius, M. (2002). The fate of clades in a world of recurrent climatic change: Milankovitch oscillations and evolution. Annual Review of Ecology and Systematics , 33(1) , 741-777.

Kavanaugh, D. H. (1985). On wing atrophy in carabid beetles (Coleoptera: Carabidae), with special reference to Nearctic Nebria. In G. E. Ball (Ed.), Taxonomy, Phylogeny and zoogeography of beetles and ants. A Volume Dedicated to the Memory of Philip Jackson Darlington, Jr.(1904-1983). Series Entomologica, 33 , 408-431.

Kavanaugh, D. H. (2015). A new species of Nebria Latreille (Insecta: Coleoptera: Carabidae: Nebriini) from the Spring Mountains of Southern Nevada. Proceedings of the California Academy of Sciences , 62 , $441-450$.

Keightley, P. D., Ness, R. W., Halligan, D. L., \& Haddrill, P. R. (2014). Estimation of the spontaneous mutation rate per nucleotide site in a Drosophila melanogaster full-sib family. Genetics ,196 , 313-320.

Keightley, P. D., Pinharanda, A., Ness, R. W., Simpson, F., Dasmahapatra, K. K., Mallet, J., Davey, J. W., \& Jiggins, C. D. (2015). Estimation of the spontaneous mutation rate in Heliconius melpomene . Molecular Biology and Evolution, 32, 239-243.

Kikvidze Z, Brooker RW, Butterfield BJ, Callaway RM, Cavieres LA, Cook BJ, Lortie CJ, Michalet R, Pugnaire FI, Xiao S, Anthelme F, Bjork RG, Cranston BH, Gavilan RG, Kanka R, Lingua E, Maalouf JP, Noroozi J, Parajuli R, Phoenix GK, Reid A, Ridenour WM, Rixen C, Schob C. 2015. The effects of foundation species on community assembly: A global study on alpine cushion plant communities. Ecology , 96 , 2064- 2069 .

Kleckova, I., Konvicka, M., \& Klecka, J. (2014). Thermoregulation and microhabitat use in mountain butterflies of the genus Erebia : importance of fine-scale habitat heterogeneity. Journal of Thermal Biology , $41,50-58$.

Knowles, L. L. (2001). Did the Pleistocene glaciations promote divergence? Tests of explicit refugial models in montane grasshopprers.Molecular Ecology , 10(3) , 691- 701.

Korneliussen, T. S., Albrechtsen, A., \& Nielsen, R. (2014). ANGSD: Analysis of Next Generation Sequencing Data. BMC Bioinformatics ,15, 356 .

Kosiński, P., Sekiewicz, K., Walas, Ł., Boratyński, A., \& Dering, M. (2019). Spatial genetic structure of the endemic alpine plantSalix serpillifolia : genetic swamping on nunataks due to secondary colonization? Alpine Botany, 129 , 107- 121.

Karney, C. F. F. (2013). Algorithms for geodesics. Journal of Geodesy , 87 , 43- 55.

Kubow, K. B., Robinson, C. T., Shama, L. N., \& Jokela, J. (2010). Spatial scaling in the phylogeography of an alpine caddisfly, Allogamus uncatus, within the central European Alps. Journal of the North American Benthological Society, 29 , 1089- 1099.

Kumar, S., Stecher, G., \& Tamura, K. (2016). MEGA7: Molecular Evolutionary Genetics Analysis Version 7.0 for Bigger Datasets. Molecular Biology and Evolution, 33 , 1870- 1874.

Leigh, J. W., \& Bryant, D. (2015). PopArt: full-feature software for haplotype network construction. Methods in Ecology and Evolution ,6 (9) , 1110- 1116.

Liu, X. (2020). Human prehistoric demography revealed by the polymorphic pattern of CpG transitions. Molecular Biology and Evolution, msaa112

Liu, X., \& Fu, Y. X. (2015). Corrigendum: Exploring population size changes using SNP frequency spectra. Nature Genetics ,47, 1099 . 
Lohse, K., Nicholls, J. A., \& Stone, G. N. (2011). Inferring the colonization of a mountain range - refugia vs. nunatak survival in high alpine ground beetles. Molecular Ecology , 20(2), 394- 408.

Merkle, E., \& You, D. (2018). nonnest2: tests of non-nested models. Retrieved 04 August, 2020, from https://cran.r-project.org/web/packages/nonnest2

Massatti, R., \& Knowles, L. L. (2014). Microhabitat differences impact phylogeographic concordance of codistributed species: Genomic evidence in montane sedges (Carex L.) from the Rocky Mountains.Evolution , $68,2833-2846$.

Nguyen, L.T., Schmidt, H. A., Von Haeseler, A., \& Minh, B. Q. (2015). IQ-TREE: a fast and effective stochastic algorithm for estimating maximum-likelihood phylogenies. Molecular Biology and Evolution ,32 , 268- 274 .

Nielsen, R., \& Wakeley, J. (2001). Distinguishing migration from isolation: a Markov chain Monte Carlo approach. Genetics ,158(2), 885- 896.

Nielsen, R., \& Slatkin, M. (2007). Likelihood analysis of ongoing gene flow and historical association. Evolution, 54(1), 44- 50 .

Oppold, A. M., \& Pfenninger, M. (2017). Direct estimation of the spontaneous mutation rate by short-term mutation accumulation lines in.Evolution Letters , 1 , 86- 92.

Pan, D., Hülber, K., Willner, W., \& Schneeweiss, G. M. (2020). An explicit test of Pleistocene survival in peripheral versus nunatak refugia in two high mountain plant species. Molecular Ecology ,29 , 172- 183.

Paun, O., Schönswetter, P., Winkler, M., Consortium, I. \& Tribsch, A. (2008), Historical divergence vs. contemporary gene flow: evolutionary history of the calcicole Ranunculus alpestris group (Ranunculaceae) in the European Alps and the Carpathians.Molecular Ecology, 17 : 4263- 4275.

Portik, D. M., Leaché, A. D., Rivera, D., Barej, M. F., Burger, M., Hirschfeld, M., Rödel, M. O., Blackburn, D. C., \& Fujita, M. K. (2017). Evaluating mechanisms of diversification in a Guineo-Congolian tropical forest frog using demographic model selection. Molecular Ecology ,26 , 5245- 5263.

Qu, Y., Zhang, R., Quan, Q., Song, G., Li, S.H. \& Lei, F. (2012). Incomplete lineage sorting or secondary admixture: disentangling historical divergence from recent gene flow in the Vinous-throated parrotbill (Paradoxornis webbianus ). Molecular Ecology ,21: 6117- 6133.

Rambaut, A. (2012). FigTree v1. 4. Available from https://beast.community/figtree

Rambaut, A., Drummond, A. J., Xie, D., Baele, G., \& Suchard, M. A. (2018). Posterior summarization in Bayesian phylogenetics using Tracer 1.7. Systematic Biology , 67, 901.

Rood, D. H., Burbank, D. W., \& Finkel, R. C. (2011). Chronology of glaciations in the Sierra Nevada, California, from ${ }^{10}$ Be surface exposure dating. Quaternary Science Reviews , 30 : 646- 661.

Rougemont, Q., \& Bernatchez, L. (2018). The demographic history of Atlantic salmon (Salmo salar ) across its distribution range reconstructed from approximate Bayesian computations. Evolution ,72 : 1261- 1277

Rovito, S. M. (2010). Lineage divergence and speciation in the Web-toed Salamanders (Plethodontidae: Hydromantes ) of the Sierra Nevada, California. Molecular Ecology , 19(20) , 4554- 4571.

Rovito, S. M., \& Schoville, S. D. (2017). Testing models of refugial isolation, colonization and population connectivity in two species of montane salamanders. Heredity (Edinb), 119 , 265- 274.

Rubidge, E. M., Patton, J. L., \& Moritz, C. (2014). Diversification of the Alpine Chipmunk, Tamias alpinus , an alpine endemic of the Sierra Nevada, California. BMC evolutionary biology ,14(1), 34 .

Schonswetter, P., \& Schneeweiss, G. M.. (2019). Is the incidence of survival in interior Pleistocene refugia (nunataks) underestimated? Phylogeography of the high mountain plant. Ecology Evolution ,9 , 4078- 4086. 
Schoville, S. D., \& Roderick, G. K. (2009). Alpine biogeography of Parnassian butterflies during Quaternary climate cycles in North America. Molecular Ecology, 18, 3471- 3485.

Schoville, S. D., \& Roderick, G. K. (2010). Evolutionary diversification of cryophilic Grylloblatta species (Grylloblattodea: Grylloblattidae) in alpine habitats of California.BMC Evolutionary Biology , 10 , 163.

Schoville, S. D., Roderick, G. K., \& Kavanaugh, D. H. (2012). Testing the 'Pleistocene species pump'in alpine habitats: lineage diversification of flightless ground beetles (Coleoptera: Carabidae: Nebria) in relation to altitudinal zonation. Biological Journal of the Linnean Society, 107, 95- 111.

Schneeweiss, G. M., \& Schoenswetter, P. (2011). A re-appraisal of nunatak survival in arctic-alpine phylogeography. Molecular Ecology , 20 (2), 190-192.

Segarra-Moragues, J. G., Palop-Esteban, M., Gonzalez-Candelas, F., \& Catalan, P. (2007). Nunatak survival vs. tabula rasa in the Central Pyrenees: a study on the endemic plant species Borderea pyrenaica(Dioscoreaceae). Journal of Biogeography, 34, 1893- 1906.

Schonswetter, P., Stehlik, I., Holderegger, R., \& Tribsch, A. (2005). Molecular evidence for glacial refugia of mountain plants in the European Alps. Molecular Ecology , 14(11), 3547- 3555.

Sim, Z., Hall, J. C., Jex, B., Hegel, T. M., \& Coltman, D. W. (2016). Genome-wide set of SNPs reveals evidence for two glacial refugia and admixture from postglacial recolonization in an alpine ungulate.Molecular Ecology , 25 , 3696- 3705.

Simon, C., Frati, F., Beckenbach, A., Crespi, B., Liu, H., \& Flook, P. (1994). Evolution, weighing, and phylogenetic utility of mitochondrial gene sequences and a compilation of conserved polymerase chain reaction primers. Annals of the Entomological Society of America ,87, 651- 701.

Starcova, M., Vohralik, V., Kryštufek, B., Bolfíková, B. Č., \& Hulva, P. (2016). Phylogeography of the Alpine shrew, Sorex alpinus(Soricidae, Mammalia). Journal of Vertebrate Biology ,65 , 107- 116.

Stehlik, I., Blattner, F. R., Holderegger, R., \& Bachmann, K. (2002). Nunatak survival of the high Alpine plant Eritrichium nanum (L.) Gaudin in the central Alps during the ice ages. Molecular Ecology , 11(10), 2027- 2036.

Stoltz, M., Bauemer, B., Bouckart, R., Fox, C., Hiscott, G., \& Bryant, D. (2019). Bayesian inference of species trees using diffusion models. arXiv preprint arXiv:1909.07276.

Theissinger, K., Bálint, M., Feldheim, K. A., Haase, P., Johannesen, J., Laube, I., \& Pauls, S. U. (2013). Glacial survival and post-glacial recolonization of an arctic-alpine freshwater insect (Arcynopteryx dichroa, Plecoptera, Perlodidae) in Europe.Journal of Biogeography , 40 , 236- 248.

Thiel-Egenter, C., Alvarez, N., Holderegger, R., Tribsch, A., Englisch, T., Wohlgemuth, T., Colli, L., Gaudeul, M., Gielly, L., Jogan, N., Linder, H.P., Negrini, R., Niklfeld, H., Pellecchia, M., Rioux, D., Schonswetter, P., Taberlet, P., van Loo, M., Winkler, M., and Gugerli, F. (2011), Break zones in the distributions of alleles and species in alpine plants. Journal of Biogeography, $38: 772-782$.

Vuong, Q. H. (1989). Likelihood ratio tests for model selection and nonnested hypotheses. Econometrica , 57(2), 307-333.

Wachter, G. A., Papadopoulou, A., Muster, C., Arthofer, W., Knowles, L. L., Steiner, F. M., \& Schlick-Steiner, B. C. (2016). Glacial refugia, recolonization patterns and diversification forces in AlpineendemicMegabunus harvestmen. Molecular Ecology , 25 , 2904- 2919.

Wallis, G. P., Waters, J. M., Upton, P., \& Craw, D. (2016). Transverse Alpine Speciation Driven by Glaciation. Trends in Ecology 83 Evolution, 31, 916-926.

Warmuth, V. M., \& Ellegren, H. (2019). Genotype-free estimation of allele frequencies reduces bias and improves demographic inference from RADSeq data. Molecular Ecology Resources , 19 , 586- 596. 
Weir, B. S., \& Cockerham, C. C. 1984. Estimating F-statistics for the analysis of population structure. Evolution, 38 , 1358- 1370.

Weng, Y. M., Yang, M. M., \& Yeh, W. B. (2016). A comparative phylogeographic study reveals discordant evolutionary histories of alpine ground beetles (Coleoptera, Carabidae). Ecology and Evolution , 6 , 20612073.

Zaman, K., Hubert, M. K., \& Schoville, S. D. (2019). Testing the role of ecological selection on colour pattern variation in the butterfly Parnassius clodius. Molecular Ecology , 28 , 5086- 5102.

\section{Data Accessibility}

All the genetic data sequenced in this study have been deposited in National Center for Biotechnology Information (NCBI) including COI gene in GenBank (accession number: MT676423-MT676429) and next generation sequences reads under BioProject (PRJNA645878).

\section{Author Contributions}

This study was conceptually designed by YMW and SDS. The data collection and analyses were conducted by YMW. All authors contributed to the writing of the manuscript, with the first draft completed by YMW as part of his dissertation.

\section{Supplementary Material}

The supplementary materials are deposited in the following file: MEC_Supplemental_Information_Weng etal.docx

\section{Figure Legends}

Figure 1. Sample sites of the Nebria ingens complex in the Sierra Nevada, California. Collecting sites of $N$. riversi, the intermediate morphotype, and $N$. ingens are depicted with solid triangles, squares, and circles, respectively. The survey sites where no populations were located (despite survey efforts) are denoted by x marks. The extent of last glacial maximum is shown as a grey line (Rood, Burbank, \& Finkel, 2011). Geographical names for each site can be found in Table 2 .

Figure 2 . The Bayesian mitochondrial COI gene tree of theNebria ingens complex with posterior probabilities listed above the nodes and divergence time listed beneath the nodes. Divergence time is estimated using a strict molecular clock (0.0113 substitutions per lineage per million years) and is denoted by the mean and $95 \%$ confidence interval. The two major clades correspond to a northern clade consisting of $N$. riversi, intermediate morphotypes, and some $N$. ingens samples, and a southern clade consisting solely of the intermediate morphotype and $N$. ingens .

Figure 3. The SNAPP species tree of individuals from eight sites using the fully-filtered SNP dataset. The thick black line shows the consensus tree, and the numbers at each node denote the posterior probability.

Figure 4 . Population structure of the $N$. ingens complex based on sNMF clustering analysis for $\mathrm{K}=6$ (left) and $\mathrm{K}=10$ (right). The concatenated ML tree is shown on the $\mathrm{K}=6$ map with bootstrap support for major nodes. The colors of population clusters and clades reflect the major drainage basins of the Sierra Nevada, and the drainage is roughly circled by the dashed lines.

Figure 5. The model accuracies estimated using conSturct. For the $\mathrm{K}=3$ to $\mathrm{K}=8$, spatial models are significantly more accurate than non-spatial models $(p<0.05)$.

Figure 6. Stairway plots of eight selected sites including site 1 (A), site 3 (B), site 9 (C), site 10 (D), site $12(\mathrm{E})$, site $17(\mathrm{~F})$, site $24(\mathrm{G})$, and site $27(\mathrm{H})$. The solid lines, dashed lines, and dotted lines represent the median, $12.5 \%$ and $87.5 \%$, and $2.5 \%$ and $97.5 \%$ confidence intervals, respectively. All sites show dramatic population declines in past 30 thousand years. 
Figure 7. Measurements of TMRCA (A) and $F_{\text {ST }}(\mathrm{B})$ for paired populations from the same (solid circle) and different (empty circle) drainages, plotted against geographical distance. For TMRCA (A), there is a significant difference between same and different drainage population pairs, with no significant correlation between TMRCA and geographical distance. The TMRCA across different drainages mostly predates the last glacial maximum (LGM), which is denoted by the grey box. For $F$ ST (B), there is no significant difference between population pairs within and among drainages, but the correlation between $F$ sT and geographic distance is significant.

Table 1. Hypothesis testing framework for competing glacial refugia hypotheses.

\begin{tabular}{lll}
\hline Model name & Periglacial refugia & Periglacial refugia \\
\hline Submodel & Submodel & single refugium \\
Hypothesis & Hypothesis & The entire species complex shares one glacial refugium, in the pe \\
Expected \# of clusters & Expected \# of clusters & $\mathrm{K}=1$ \\
sNMF & sNMF & unsupported \\
spatial-model of conStruct & spatial-model of conStruct & unsupported \\
AIC (TMRCA) & AIC (TMRCA) & $N A$ \\
BIC (TMRCA) & BIC (TMRCA) & $N A$ \\
Model comparison & Model comparison & $N A$ \\
\hline
\end{tabular}

$+\mathrm{K}=5$ has same set of paired population due to the lack of samples from Merced drainage.

Table2. Collecting information for each sample site.

\begin{tabular}{lllllll}
\hline Code & Site name & Drainage system & Sample size (nuclear) & Sample size (mtDNA) & Latitude & Longitud \\
1 & Conness Lake & Tuolumne & 32 & 22 & 37.94329 & -119.2861 \\
2 & Kuna Lake & Tuolumne & 0 & 1 & 37.84874 & -119.2592 \\
3 & Donohue Pass & Merced/San Joaquin & 16 & 16 & 37.75289 & -119.2484 \\
4 & Lyell Peak & Merced/San Joaquin & 16 & 15 & 37.74956 & -119.2599 \\
5 & Ritter Range & San Joaquin & 0 & 0 & 37.71442 & -119.2035 \\
6 & Ottoway Lake & Merced & 0 & 3 & 37.63967 & -119.3971 \\
7 & Recess Lakes & San Joaquin & 3 & 10 & 37.43124 & -118.7846 \\
8 & Ruby Lake & San Joaquin & 16 & 12 & 37.41155 & -118.7739 \\
9 & Italy Lake & San Joaquin & 10 & 10 & 37.34084 & -118.7673 \\
10 & Selden Pass & San Joaquin & 14 & 17 & 37.28632 & -118.8853 \\
11 & Piute Pass & San Joaquin & 22 & 18 & 37.23683 & -118.6799 \\
12 & Lamarck Lakes & San Joaquin & 17 & 20 & 37.20853 & -118.6511 \\
13 & Hungry Packer Lake & San Joaquin/Kings & 11 & 11 & 37.16094 & -118.6407 \\
14 & Treasure Lake & Kings & 9 & 11 & 37.14502 & -118.5800 \\
15 & Sam Mack Lake & Kings & 14 & 6 & 37.11192 & -118.5117 \\
16 & Dusy Pass & Kings & 2 & 13 & 37.09534 & -118.5342 \\
17 & Taboose Pass & Kings & 9 & 5 & 36.97993 & -118.3987 \\
18 & Sixty Lakes & Kings & 4 & 2 & 36.82479 & -118.4338 \\
19 & Sphinx Lakes & Kings/Kern & 2 & 12 & 36.71427 & -118.5148 \\
20 & Milly's Footpass & Kings/Kern & 12 & 4 & 36.69039 & -118.4345 \\
21 & North Forester & Kings/Kern & 4 & 25 & 36.68882 & -118.3764 \\
22 & South Forester & Kern & 16 & 16 & 36.63116 & -118.3448 \\
23 & Wright Lakes & Kern & 12 & 14 & 36.59707 & -118.6658 \\
24 & Pear Lake & Kaweah & 12 & 14 & 36.59707 & -118.6658 \\
25 & Crabtree Lakes & Kern & 14 & 6 & 36.54445 & -118.3236 \\
26 & Monarch Lake & Kaweah/Kern & 6 & & 36.50159 & -118.2471 \\
& & & & & &
\end{tabular}


Table3. Successful copulation rate of $N$. riversi, intermediate morphotypes, and $N$. ingens

\begin{tabular}{llll}
\hline & $N$. riversi male & intermediate male & $N$. ingens male \\
$N$. riversi female & $3 / 3$ & $7 / 7$ & $1 / 5$ \\
intermediate female & $4 / 4$ & $6 / 7$ & $0 / 5$ \\
$N$. ingens female & $1 / 3$ & $6 / 7$ & $1 / 5$ \\
\hline
\end{tabular}

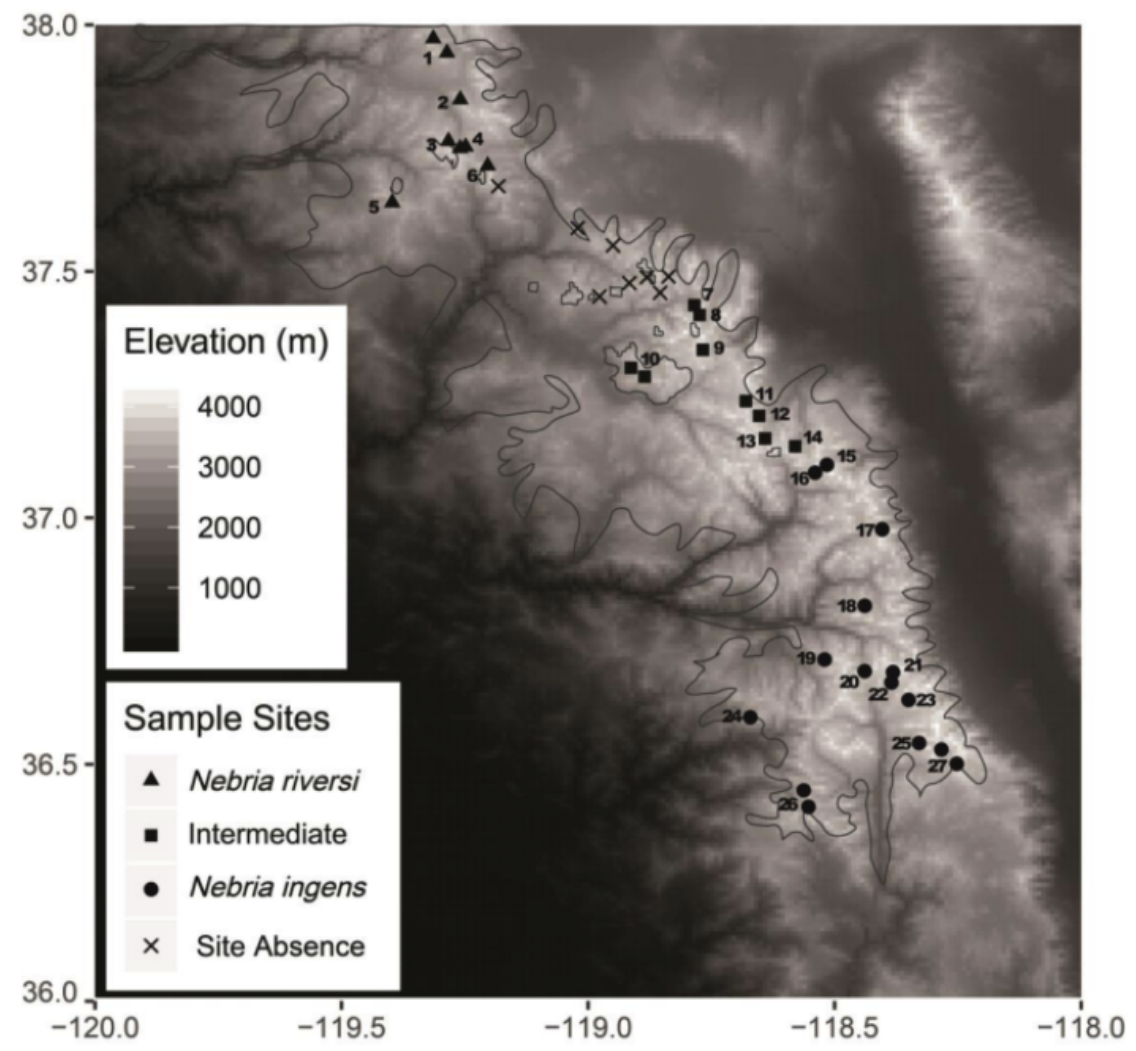

\section{Hosted file}

Figures-new.pdf available at https://authorea.com/users/355026/articles/478442-drainagebasins-serve-as-multiple-glacial-refugia-for-alpine-habitats-in-the-sierra-nevadamountains-california 